\title{
Diagnostic Utility of PAX8 and PAX2 Immunohistochemistry in the Identification of Metastatic Müllerian Carcinoma in Effusions
}

\author{
William Wiseman, D.O., Claire W. Michael, M.D., and Michael H. Roh, M.D., Ph.D.*
}

\begin{abstract}
Morphologic distinction of Müllerian carcinomas from non-Müllerian carcinomas in effusion specimens by cytomorphology alone can be diagnostically challenging. Therefore, immunohistochemical adjuncts can be useful in differentiating Müllerian from non-Müllerian metastases. In this study, we evaluated the expression of PAX8 and PAX2 in malignant effusions collected from patients with known Müllerian and non-Müllerian carcinomas. Sections from cell blocks prepared from 152 effusion specimens (54 and 98 cases representing metastases from Müllerian and non-Müllerian primaries, respectively) were immunostained with rabbit polyclonal antibodies against PAX8 and PAX2. Immunopositivity was defined as the presence of strong nuclear staining in at least $25 \%$ of the tumor cells. Fifty-two (96\%) and 13 (24\%) of the 54 Müllerian carcinomas were positive for PAX8 and PAX2, respectively. PAX8 positivity was seen in only four (4\%) of 98 non-Müllerian carcinomas; these represented metastasis from a large cell neuroendocrine lung carcinoma, papillary thyroid carcinoma, renal cell carcinoma, and acinic cell carcinoma of the parotid gland. PAX2 positivity was not seen in any of the non-Müllerian carcinomas. The results demonstrate that both PAX8 and PAX2 are highly specific markers for metastatic Müllerian carcinomas in cell block preparations from effusion specimens $196 \%$ and $100 \%$, respectively). PAX8, however, is more sensitive than PAX2 in identifying Müllerian carcinomas in fluids (96\% versus 24\%). Overall, immunohistochemistry for PAX8 and PAX2 represent diagnostically useful adjuncts in identifying a Müllerian carcinoma as a source of a malignant effusion. Diagn. Cytopathol. 2011;39:651-656. (c) 2010 Wiley-Liss, Inc.
\end{abstract}

Key Words: PAX8; PAX2; Müllerian; carcinoma; effusion

Department of Pathology, University of Michigan Medical School, Ann Arbor, Michigan

*Correspondence to: Michael H. Roh, M.D., Ph.D., Department of Pathology, University of Michigan Medical School, 2G332 UH, 1500 E. Medical Center Drive, Ann Arbor, Michigan 48109-5054.

E-mail: mikro@med.umich.edu

Received 10 March 2010; Accepted 17 April 2010

DOI $10.1002 / \mathrm{dc} .21442$

Published online 14 October 2010 in Wiley Online Library (wileyonlinelibrary.com).
Metastatic carcinoma represents a common cause of fluid accumulation in the pleural, peritoneal, and pericardial spaces. In some cases, there is a known cancer history at the time a malignant effusion is diagnosed. Nonetheless, in other cases, a malignant effusion is diagnosed in the absence of a known primary malignancy. ${ }^{1}$ Occasionally, cytologic evaluation of an effusion specimen can uncover the presence of a distinct primary in the setting of a previously documented cancer history. It is therefore important to determine or confirm a metastasis from a particular primary site as this will yield clinically useful information about the nature and extent of disease and facilitate the tailoring of optimal treatment regimens. ${ }^{2}$ Because of overlapping cytomorphologic features of carcinomatous effusions by various primaries, ${ }^{3}$ confident identification of a primary can be difficult by cytomorphologic evaluation alone. Therefore, immunohistochemistry is often employed to gain insight into the primary site. ${ }^{4-6}$

Malignancies of the female gynecological (Müllerian) tract often present initially as ascites and effusions. The accurate diagnosis of malignant effusions resulting from metastatic Müllerian carcinomas is essential as specific chemotherapeutic regimens are used to treat distinct Müllerian carcinomas. For instance, high-grade serous carcinomas are typically treated with combination chemotherapy consisting of carboplatin and paclitaxel. ${ }^{7}$ Furthermore, because these cancers usually present at an advanced stage, neoadjuvant chemotherapy with subsequent debulking surgery can be considered as a treatment option in cases where the extent of disease is deemed unresectable at initial presentation. ${ }^{8}$ Also, the development of an effusion can indicate recurrence of malignancy after treatment. In both contexts, cytologic evaluation of effusions represents a minimally invasive method to obtain a specific pathologic diagnosis; accurately diagnosing a metastasis from a Müllerian carcinoma in effusions can lead to appropriate, prompt treatment. 
The most common Müllerian carcinoma to present in effusions is serous carcinoma which is known to originate in the ovary, fallopian tube, peritoneum, and endometrium, ${ }^{9}$ the majority of which are high-grade and manifest as single cells or small clusters in fluids. This cytomorphologic presentation can overlap with carcinomas originating from other primary sites such as breast, lung, and pancreas. ${ }^{3,10}$ Furthermore, in some cases, individual tumor cells can be difficult to distinguish from reactive mesothelial cells. Thus, especially in cases where the primary site of malignancy is unknown, diagnosing a malignant effusion and subsequently distinguishing a Müllerian from a non-Müllerian malignancy with certainty by cytomorphology alone can be challenging.

Immunohistochemical adjuncts have been previously reported in the diagnosis of Müllerian carcinomas. Notably, high-grade serous carcinomas are often immunoreactive for $\mathrm{p} 53^{11}$ and WT-1. ${ }^{12,13}$ However, mesothelial cells are also immunoreactive for $\mathrm{WT}-1$ and reactive mesothelium can occasionally be $\mathrm{p} 53$-positive ${ }^{14}$ thereby rendering immunohistochemical stains for WT-1 and p53 difficult to interpret in some cases. Furthermore, immunoreactivity for ER and PR can support Müllerian origin; however, positivity for these hormone receptors can often be seen in the context of malignant effusions secondary to metastatic breast carcinomas. ${ }^{15}$

Recently the transcription factors, PAX2 and PAX8, have been reported to be expressed in Müllerian-derived epithelium such as the fallopian tube epithelium. ${ }^{16-18}$ Furthermore, studies have shown that their expression is maintained in malignant tumors derived from this epithelium. For instance, Roh et al. have reported strong, diffuse PAX8 expression in the vast majority of serous carcinomas. ${ }^{19}$ Furthermore, Tong et al. also reported that PAX2 is positive in a significant proportion of Müllerian carcinomas. ${ }^{18}$ In addition, Nonaka et al. and Chivukula et al. investigated the utility of PAX8 and PAX2, respectively, to differentiate ovarian carcinomas from breast carcinomas in surgically resected specimens. ${ }^{20,21}$

To date, there are no reports that investigate the utility of PAX2 and PAX8 immunohistochemistry in effusion specimens. Therefore, we sought to investigate the diagnostic utility of immunohistochemistry for PAX2 and PAX8 as adjuncts to cytology in the identification and differentiation of Müllerian carcinomas from non-Müllerian carcinomas in effusions.

\section{Methods}

After approval was obtained from the Institutional Review Board at University of Michigan, the electronic pathology database along with surgical pathology reports, clinical notes, and radiology reports were examined to identify patients in whom carcinomatous effusions (pleural, peritoneal, and pericardial) from a known primary site were
Table I. Primary Sites of Malignancy in the 152 Patients With Carcinomatous Effusions

\begin{tabular}{|c|c|c|c|}
\hline \multirow{2}{*}{$\frac{\text { Primary tumor }}{\text { Ovary/fallopian tube }}$} & \multicolumn{3}{|c|}{ \# Patients \# PAX8(+) \# PAX2(+) } \\
\hline & & & \\
\hline Serous carcinoma & 34 & 32 & 7 \\
\hline Carcinosarcoma & 2 & 2 & 0 \\
\hline Clear cell carcinoma & 2 & 2 & 1 \\
\hline Endometrioid & 1 & 1 & 0 \\
\hline Poorly differentiated carcinoma & 3 & 3 & 1 \\
\hline \multicolumn{4}{|l|}{ Primary Peritoneal } \\
\hline Serous carcinoma & 7 & 7 & 2 \\
\hline Poorly differentiated carcinoma & 1 & 1 & 1 \\
\hline \multicolumn{4}{|l|}{ Endometrium } \\
\hline Serous carcinoma & 3 & 3 & 0 \\
\hline Carcinosarcoma & 1 & 1 & 1 \\
\hline \multicolumn{4}{|l|}{ Lung } \\
\hline Adenocarcinoma & 20 & 0 & 0 \\
\hline Squamous cell carcinoma & 3 & 0 & 0 \\
\hline Non-small cell carcinoma & 8 & 0 & 0 \\
\hline Large cell neuroendocrine carcinoma & 2 & 1 & 0 \\
\hline Small cell carcinoma & 1 & 0 & 0 \\
\hline \multicolumn{4}{|l|}{ Breast } \\
\hline Ductal carcinoma & 25 & 0 & 0 \\
\hline Lobular carcinoma & 2 & 0 & 0 \\
\hline Colloid carcinoma & 1 & 0 & 0 \\
\hline \multicolumn{4}{|l|}{ Gastroesophageal } \\
\hline Adenocarcinoma & 15 & 0 & 0 \\
\hline \multicolumn{4}{|l|}{ Pancreaticobiliary } \\
\hline Adenocarcinoma & 9 & 0 & 0 \\
\hline \multicolumn{4}{|l|}{ Colon } \\
\hline Adenocarcinoma & 6 & 0 & 0 \\
\hline \multicolumn{4}{|l|}{ Appendix } \\
\hline Adenocarcinoma & 1 & 0 & 0 \\
\hline \multicolumn{4}{|l|}{ Larynx } \\
\hline Adenoid cystic carcinoma & 1 & 0 & 0 \\
\hline \multicolumn{4}{|l|}{ Bladder } \\
\hline Urothelial cell carcinoma & 1 & 0 & 0 \\
\hline \multicolumn{4}{|l|}{ Thyroid } \\
\hline Papillary thyroid carcinoma & 1 & 1 & 0 \\
\hline \multicolumn{4}{|l|}{ Kidney } \\
\hline Renal cell carcinoma, clear cell type & 1 & 1 & 0 \\
\hline \multicolumn{4}{|l|}{ Parotid } \\
\hline Acinic cell carcinoma & 1 & 1 & 0 \\
\hline
\end{tabular}

diagnosed from 2000 to 2003 . The slides prepared from each effusion specimen were reviewed and cases in which sufficient tumor was present in the formalin-fixed, paraffin-embedded cell block preparations were identified by examining recut, Hematoxylin and Eosin (H\&E) stained slides. A total of 152 malignant effusions, one from each patient, were included in this study (Table I).

Immunohistochemistry was performed using the DAKO Autostainer (DAKO, Carpinteria, CA) using DAKO $\mathrm{LSAB}+$ and 3,3'-diaminobenzidine as the chromogen. For each case, 4- $\mu \mathrm{m}$ unstained sections were prepared from the cell blocks, deparaffinized, and immunostained after antigen retrieval in $0.01 \mathrm{M}$ citrate buffer, $\mathrm{pH} 6.0$ (DAKO, Carpinteria, CA). Immunohistochemistry was performed using the rabbit polyclonal anti-PAX2 (1:100 dilution; Invitrogen, Camarillo, CA) and rabbit polyclonal antiPAX8 (1:200 dilution; Protein Tech, Chicago, IL) antibodies along with appropriate controls. The EnVision+ System for use with rabbit primary antibodies (DAKO, 
Carpinteria, CA) was used as the secondary antibody. Each immunostained slide was reviewed blindly by two reviewers (WW and MHR) and strong nuclear staining for PAX2 and PAX8 in at least $25 \%$ of the tumor cells was considered as a positive result. The presence or absence of staining of background mesothelial cells and histiocytes was also recorded in each case.

\section{Results}

A total of 152 patients with metastatic carcinomatous effusions from a known primary site were identified and represented the source of material utilized in this study (Table I). Fifty-four (36\%) patients were diagnosed with a malignant effusion secondary to a Müllerian primary malignancy (ovary, fallopian tube, primary peritoneal, and endometrium); the majority of these (44 of $54 ; 81 \%$ ) represented high-grade serous carcinomas. The remainder of the Müllerian primary malignancies consisted of: three carcinosarcomas; two clear cell carcinomas; one highgrade endometrioid carcinoma; and four poorly differentiated carcinomas. The lung represented the most common non-Müllerian primary tumor that metastasized to fluids (34 of 152; 22\%): 20 adenocarcinomas; three squamous cell carcinomas; eight non-small cell carcinomas; two large cell neuroendocrine carcinomas; and one small cell carcinoma. Breast primaries represented the next most frequent non-Müllerian primary source of malignant effusions (28 of 152; 18\%): 25 ductal carcinomas, two lobular carcinomas, and one colloid carcinoma. In 15 (10\%), nine (6\%), six $(4 \%)$, and one case $(1 \%)$, the malignant effusions were secondary to metastases from upper gastrointestinal, pancreaticobiliary, colonic, and appendiceal primaries, respectively. The remaining 5 of 152 (3\%) primary malignancies to give rise to carcinomatous effusions consisted of: papillary thyroid carcinoma; renal cell carcinoma, clear cell type; urothelial cell carcinoma; adenoid cystic carcinoma; and acinic cell carcinoma. Results for PAX8 and PAX2 immunohistochemistry for the cases are summarized in Table I.

Immunohistochemistry revealed strong nuclear immunoreactivity for PAX8 in at least $25 \%$ of the tumor cells in 56 cases. Specifically, over $50 \%$ of the tumor cells were positive in 52 cases and between $25 \%$ and $50 \%$ of the tumor cells were positive in the remaining four. Fifty-two of the PAX8(+) malignant effusions represented metastases from Müllerian primaries (Table II), which included serous carcinomas, carcinosarcomas, clear cell carcinomas, and the one case of endometrioid carcinoma (Fig. 1). In one additional case of serous carcinoma, only rare tumor cells $(\sim 1 \%$ of the total tumor cell population) were highlighted by the PAX8 immunostain; this case was scored as negative, however, as the vast majority of the tumor cells were negative for PAX8. Of note, in each of the carcinosarcoma cases, the sarcomatous component was not present in the malignant effusion. Overall, the sensitivity for PAX8 in detecting Müllerian
Table II. Sensitivity and Specificity of PAX8 and PAX2 as Immunohistochemical Markers for Müllerian Carcinomas in Effusions

\begin{tabular}{lcc}
\hline & $P A X 8(+)$ & $P A X 2(+)$ \\
\hline Müllerian Carcinoma $(n=54)$ & $52(96 \%)$ & $13(24 \%)$ \\
Non-Müllerian Carcinoma $(n=98)$ & $4(4 \%)$ & $0(0 \%)$ \\
Sensitivity & $96 \%$ & $24 \%$ \\
Specificity & $96 \%$ & $100 \%$ \\
\hline
\end{tabular}

malignancies in fluids was $96 \%$ (52/54). The four remaining PAX8(+) cases represented malignant effusions secondary to non-Mullerian primaries including: a large cell neuroendocrine carcinoma of the lung; papillary thyroid carcinoma; and an acinic cell carcinoma of the parotid gland; and a renal cell carcinoma, clear cell type (Fig. 2). In the remaining nonMullerian carcinomas examined, none of the tumor cells were positive for PAX8. Overall, four of the 98 non-Müllerian malignant effusions were positive for PAX8; thus, the specificity of PAX8 for identifying Müllerian malignancies was $96 \%$ (Table II).

Next, immunohistochemistry for PAX2 revealed strong nuclear PAX2 immunoreactivity in 13 cases (Table I). All 13 cases were malignant effusions secondary to Müllerian primaries (Table II). In eight cases, over half of the tumor cells were highlighted by PAX2 and $25-50 \%$ of the tumor cells were $\operatorname{PAX} 2(+)$ in the remaining five. The remainder of the Müllerian carcinomas and all of the non-Müllerian carcinomas were completely negative for PAX2. Overall, the sensitivity for PAX2 in detecting Müllerian malignancies in effusions was $24 \%$ (13/54). Because none of the non-Müllerian tumors were PAX2(+), the specificity of PAX2 for identifying Müllerian malignancies in fluids was $100 \%$. Of note, all $13 \operatorname{PAX} 2(+)$ tumors were also PAX8(+). Finally, in every case, PAX8 and PAX2 immunohistochemistry failed to highlight the background mesothelial cells and histiocytes in this study (Fig. 1).

\section{Discussion}

Recently, the expression of the transcription factors, PAX2 and PAX8, have been reported in carcinomas of the female genital tract including serous, endometrioid, and clear cell carcinomas. ${ }^{18,20,21}$ As there are no studies to date that have investigated the application of PAX2 and PAX8 immunohistochemistry to effusions, we sought to determine the expression of these two markers in Müllerian carcinomas in fluid specimens. In this study, we discovered that the majority of metastatic carcinomas of Müllerian origin were immunoreactive for PAX8 in effusions with high sensitivity (96\%). Specifically, in all but two serous carcinomas and in all of the metastatic carcinomas originating from carcinosarcomas, clear cell carcinomas, and endometrioid carcinoma, PAX8 immunoreactivity was seen in at least $25 \%$ of the tumor cells. This is concordant with the findings by Bowen et al. which showed a high frequency of PAX8 immunoreactivity in 


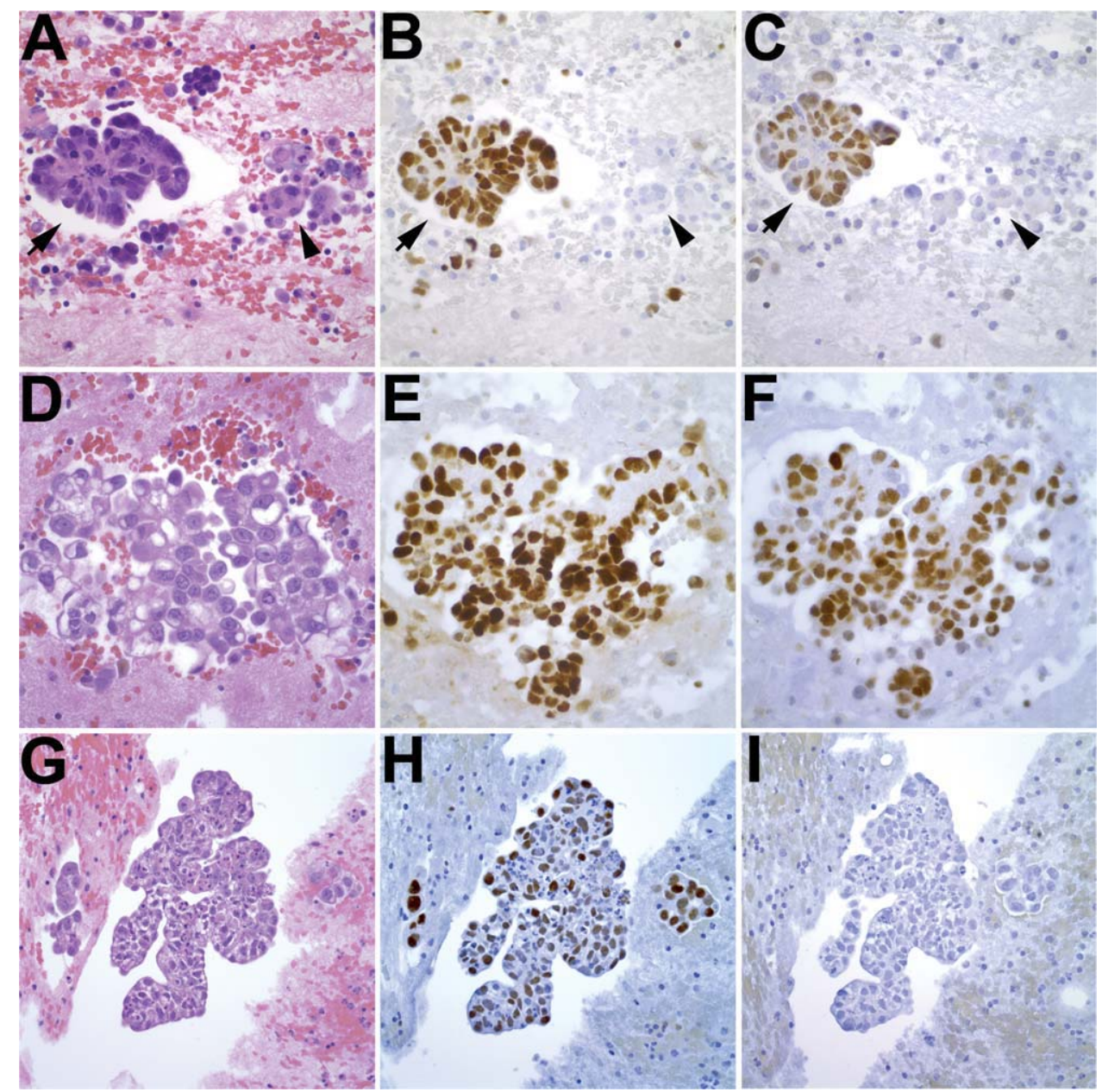

Fig. 1. PAX8 and PAX2 highlights metastatic Müllerian carcinomas in effusions. A-C: Metastatic serous carcinoma in cell block sections of pleural fluid, $\times 600$. D-F: Metastatic clear cell carcinoma in cell block sections of peritoneal fluid, $\times 600$. G,H: Metastatic endometrioid carcinoma in a cell block prepared from a malignant peritoneal effusion, $\times 400$. H\&E stained sections are shown in A, D, and G, PAX8 immunostains in B, E, and H, and PAX2 immunostains in C, F, and I. B,C: Strong nuclear positivity for PAX8 and PAX2 are observed in the serous carcinoma cluster on the left indicated by the arrow; the background mesothelial cells are not immunoreactive for PAX8 and PAX2. [Color figure can be viewed in the online issue, which is available at wileyonlinelibrary.com.]

various subtypes of ovarian carcinomas. ${ }^{16}$ Furthermore, our results are complementary to those reported by Davidson et al. who applied gene expression profiling to malignant effusions; they reported increased gene expression of PAX8 in serous carcinomas in fluid specimens. ${ }^{22}$

Next, we sought to determine whether PAX8 immunopositivity was specific for Müllerian carcinomas by examining the expression of PAX8 in non-Müllerian carcinomatous effusions in parallel. Lung, breast, gastrointestinal, and pancreaticobiliary carcinomas represent common etiologies for malignant effusions. ${ }^{3}$ We found that $100 \%$ of breast, gastrointestinal, and pancreaticobiliary carcinomas and $97 \%$ of lung carcinomas in our study were negative for PAX8. Of note, all 20 of the lung adenocarcinomas, the subtype of lung carcinoma most likely to mimic Müllerian adenocarcinoma, were not immunoreactive for PAX8. These results are consistent with prior reports by
Nonaka and colleagues that PAX8 is a highly specific marker for distinguishing Müllerian carcinomas from these non-Müllerian carcinomas. ${ }^{20,23}$ PAX8 immunoreactivity in effusions was seen in only four non-Müllerian carcinomas including metastatic papillary thyroid carcinoma and renal cell carcinoma. This can be reconciled in light of the finding that PAX8 is expressed in thyroid follicular epithelium and renal tubular epithelium as well as thyroid carcinomas and renal cell carcinomas. ${ }^{23,24}$ It should be noted that carcinomas of thyroid and renal origin rarely metastasize to fluids ${ }^{10}$ explaining the low number of these malignant effusions in our study population; nonetheless, our results confirm that PAX8 can be useful in confirming the presence of metastatic carcinomas from the thyroid and kidney in effusions. We also found one case each of a large cell neuroendocrine lung carcinoma and an acinic cell carcinoma of the salivary gland to be 


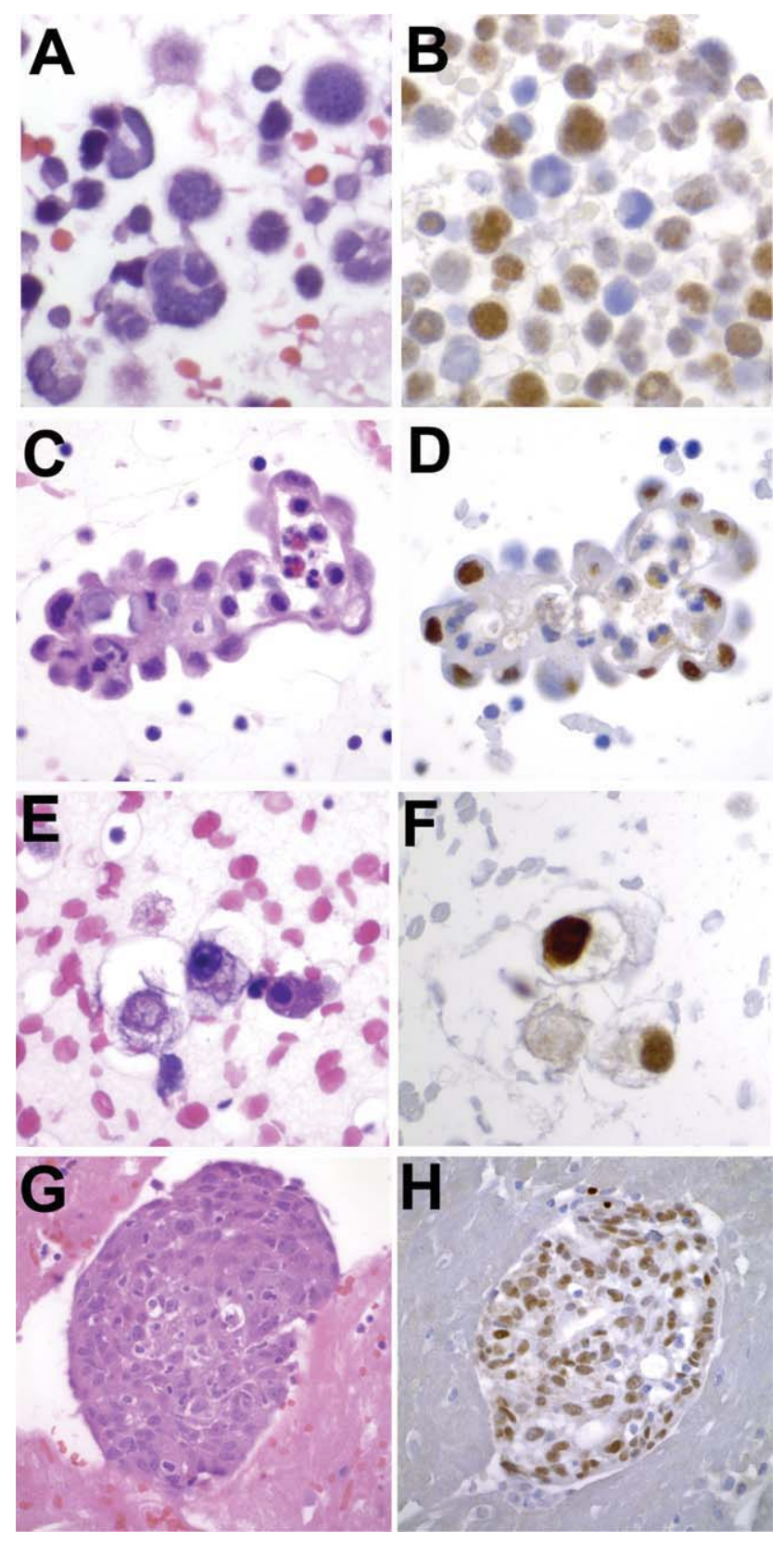

Fig. 2. PAX8 immunoreactivity in the four metastatic non-Müllerian carcinomatous effusions. A, B: Large cell neuroendocrine carcinoma in a pleural effusion, $\times 600$. C, D: Papillary thyroid carcinoma metastatic to a pleural effusion, $\times 600$. E, F: Renal cell carcinoma in a pleural effusion, $\times 600$. G, H: Acinic cell carcinoma metastatic to pleural fluid, $\times 400$. $\mathrm{H} \& \mathrm{E}$ stained sections are shown in A, C, E, and G. PAX-8 immunostains are shown in B, D, F, and H. [Color figure can be viewed in the online issue, which is available at wileyonlinelibrary.com.]

immunoreactive for PAX8. These also represent malignancies that seldomly metastasize to effusions and because the expression of PAX8 in these malignancies have not been studied in detail, additional studies will be required to determine the frequency of PAX8 positivity in these cancers. Nonetheless, our results overall demonstrate that PAX8 is a highly sensitive and specific marker for metastatic carcinomas of Müllerian origin in fluids.

We sought to ascertain the sensitivity and specificity of PAX2 as a marker for Müllerian carcinomas. In contrast to PAX8, PAX2 was only positive in $24 \%$ of the Müllerian carcinomas in this study. This contrasts with the findings by Tong et al. who showed that over half of serous carcinomas are immunoreactive for PAX2. ${ }^{18}$ Nonetheless, our results can be reconciled in light of the more recent finding that the proportion of high-grade serous carcinomas that express PAX2 at the mRNA and protein level is significantly less than that of low grade serous carcinomas. ${ }^{25}$ It should be noted that none of the serous carcinomas in our study were low grade. Of note, PAX2 expression has been found in $61-85 \%$ of renal cell carcinomas. $^{26,27}$ Although our one case of metastatic renal cell carcinoma was negative for PAX2, it is likely that others may be PAX2 positive.

Finally, the cytomorphologic patterns of malignant mesothelioma in effusions can mimic those of carcinomas, especially those of Müllerian origin. Although the expression of PAX8 in mesothelioma is not well characterized, the majority of mesotheliomas appear to be negative for PAX2. Specifically, one previous study demonstrated that only two of 54 mesothelioma cases were immunoreactive for PAX2. ${ }^{18}$ Although additional studies examining the expression of PAX8 and PAX2 in mesotheliomas are needed, a panel of immunostains utilizing other antibodies such as those directed against calretinin, MOC-31, BerEP4, B72.3, and Leu-M1 can be helpful in distinguishing Müllerian carcinomas from mesotheliomas in effusions. ${ }^{6}$

In conclusion, our study demonstrates that both PAX8 and PAX2 are specific markers for identifying metastatic Müllerian carcinomas in effusions $(96 \%$ and $100 \%$, respectively). PAX8, however, is a more sensitive marker for Müllerian carcinomas than PAX2 (96\% versus 24\%). Nonetheless, we demonstrate the diagnostic utility of PAX8 and PAX2 in identifying and distinguishing carcinomatous effusions of Müllerian origin from those of non-Müllerian origin.

\section{Acknowledgments}

The authors thank Nancy McAnsh and Alan Burgess in the University of Michigan Comprehensive Cancer Center Research Histology and Immunoperoxidase Laboratory for their technical expertise and assistance in performing immunohistochemistry. We would also like to acknowledge support from the Anatomic Pathology Project Fund at the University of Michigan.

\section{References}

1. Monte SA, Ehya H, Lang WR. Positive effusion cytology as the initial presentation of malignancy. Acta Cytol 1987;31:448-452. 


\section{WISEMAN ET AL.}

2. Pavlidis N, Briasoulis E, Hainsworth J, Greco FA. Diagnostic and therapeutic management of cancer of an unknown primary. Eur J Cancer 2003;39:1990-2005.

3. Pereira TC, Saad RS, Liu Y, Silverman JF. The diagnosis of malignancy in effusion cytology: A pattern recognition approach. Adv Anat Pathol 2006;13:174-184.

4. Brown RW, Campagna LB, Dunn JK, Cagle PT. Immunohistochemical identification of tumor markers in metastatic adenocarcinoma. A diagnostic adjunct in the determination of primary site. Am J Clin Pathol 1997;107:12-19.

5. Cangi MG, Pecciarini L, Doglioni C. The role of immunohistochemistry in the differential diagnosis of serous effusions. Adv Clin Path 2000;4:183-185.

6. Wick MR, Moran CA, Mills SE, Suster S. Immunohistochemical differential diagnosis of pleural effusions, with emphasis on malignant mesothelioma. Curr Opin Pulm Med 2001;7:187-192.

7. Marchetti C, Pisano C, Facchini G, et al. First-line treatment of advanced ovarian cancer: Current research and perspectives. Expert Rev Anticancer Ther 2010;10:47-60.

8. Bilici A, Salepci T, Dane F, et al. Neoadjuvant chemotherapy followed by interval cytoreductive surgery in patients with unresectable, advanced stage epithelial ovarian cancer: A single centre experience. Arch Gynecol Obstet 2009.

9. Tavassoli FA, Deville P, International Agency for Research on Cancer. World Health Organization. Pathology and genetics of tumours of the breast and female genital organs. World Health Organization classification of tumours. Lyon Oxford: International Agency for Research on Cancer: Oxford University Press; 2003. p 432.

10. Bedrossian CWM. Malignant effusions: A multimodal approach to cytologic diagnosis. New York: Igaku-Shoin; 1994. xi, 275 p.

11. Bar JK, Harlozinska A, Popiela A, Noga L. Expression and mutation of p53 in tumor effusion cells of patients with ovarian carcinoma: Response to cisplatin-based chemotherapy. Tumour Biol 2001;22:83-91.

12. Han L, Pansare V, Al-Abbadi M, Husain M, Feng J. Combination of MUC5ac and WT-1 immunohistochemistry is useful in distinguishing pancreatic ductal carcinoma from ovarian serous carcinoma in effusion cytology. Diagn Cytopathol 2009;38:333-336.

13. Zhu W, Michael CW. WT1, monoclonal CEA. TTF1, and CA125 antibodies in the differential diagnosis of lung, breast, and ovarian adenocarcinomas in serous effusions. Diagn Cytopathol 2007;35: 370-375.

14. Chang MC, Cibas ES, Crum CP, Kindelberger DW. High-grade and low-grade pelvic serous neoplasms demonstrate differential p53 immunoreactivity in peritoneal washings. Cancer Cytopathol 2008;114:353-354.
15. Lee BH, Hecht JL, Pinkus JL, Pinkus GS. WT1, estrogen receptor, and progesterone receptor as markers for breast or ovarian primary sites in metastatic adenocarcinoma to body fluids. Am J Clin Pathol 2002; 117:745-750.

16. Bowen NJ, Logani S, Dickerson EB, et al. Emerging roles for PAX8 in ovarian cancer and endosalpingeal development. Gynecol Oncol 2007; 104:331-337.

17. Rabban JT, McAlhany S, Lerwill MF, Grenert JP, Zaloudek CJ. PAX2 Distinguishes benign mesonephric and mullerian glandular lesions of the cervix from endocervical adenocarcinoma. Including minimal deviation adenocarcinoma. Am J Surg Pathol 2010;34:137146.

18. Tong GX, Chiriboga L, Hamele-Bena D, Borczuk AC. Expression of PAX2 in papillary serous carcinoma of the ovary: Immunohistochemical evidence of fallopian tube or secondary Mullerian system origin? Mod Pathol 2007;20:856-863.

19. Roh MH, Kindelberger D, Crum CP. Serous tubal intraepithelial carcinoma and the dominant ovarian mass clues to serous tumor origin? Am J Surg Pathol 2009;33:376-383.

20. Nonaka D, Chiriboga L, Soslow RA. Expression of pax 8 as a useful marker in distinguishing ovarian carcinomas from mammary carcinomas. Am J Surg Pathol 2008;32:1566-1571.

21. Chivukula M, Dabbs DJ, O’Connor S, Bhargava R. PAX 2: A novel mullerian marker for serous papillary carcinomas to differentiate from micropapillary breast carcinoma. Int $\mathrm{J}$ Gynecol Pathol 2009;28:570-578.

22. Davidson B, Stavnes HT, Holth A, et al. Gene expression signatures differentiate ovarian/peritoneal serous carcinoma from breast carcinoma in effusions. J Cell Mol Med 2010.

23. Nonaka D, Tang Y, Chiriboga L, Rivera M, Ghossein R. Diagnostic utility of thyroid transcription factors Pax8 and TTF-2 (FoxE1) in thyroid epithelial neoplasms. Mod Pathol 2008;21:192-200.

24. Tong GX, Yu WM, Beaubier NT, et al. Expression of PAX8 in normal and neoplastic renal tissues: An immunohistochemical study. Mod Pathol 2009;22:1218-1227.

25. Tung CS, Mok SC, Tsang YT, et al. PAX2 expression in low malignant potential ovarian tumors and low-grade ovarian serous carcinomas. Mod Pathol 2009;22:1243-1250.

26. Gokden N, Gokden M, Phan DC, McKenney JK. The utility of PAX-2 in distinguishing metastatic clear cell renal cell carcinoma from its morphologic mimics: an immunohistochemical study with comparison to renal cell carcinoma marker. Am J Surg Pathol 2008;32:1462-1467.

27. Gokden N, Kemp SA, Gokden M. The utility of Pax-2 as an immunohistochemical marker for renal cell carcinoma in cytopathology. Diagn Cytopathol 2008;36:473-477. 